\title{
Nonadiabatic transitions in electrostatically trapped ammonia molecules
}

\author{
Moritz Kirste, ${ }^{1}$ Boris G. Sartakov, ${ }^{2}$ Melanie Schnell, ${ }^{1, *}$ and Gerard Meijer ${ }^{1}$ \\ ${ }^{1}$ Fritz-Haber-Institut der Max-Planck-Gesellschaft, Faradayweg 4-6, D-14195 Berlin, Germany \\ ${ }^{2}$ General Physics Institute, RAS, Vavilov Street 38, 119991 Moscow, Russia
}

(Received 25 November 2008; published 27 May 2009)

\begin{abstract}
Nonadiabatic transitions are known to be major loss channels for atoms in magnetic traps but have thus far not been experimentally reported upon for trapped molecules. We have observed and quantified losses due to nonadiabatic transitions for three isotopologues of ammonia in electrostatic traps by comparing the trapping times in traps with a zero and a nonzero electric field at the center. Nonadiabatic transitions are seen to dominate the overall loss rate even for the present samples that are at relatively high temperatures of $30 \mathrm{mK}$. It is anticipated that losses due to nonadiabatic transitions in electric fields are omnipresent in ongoing experiments on cold molecules.
\end{abstract}

DOI: 10.1103/PhysRevA.79.051401

PACS number(s): 37.10.Pq, 31.50.Gh, 37.10.Mn

The recent development of a large variety of methods and devices for the manipulation and trapping of neutral polar molecules offers new opportunities for molecular physics experiments [1]. Decelerated beams and trapped samples of polar molecules can be used to study intrinsic molecular properties, such as energy level splittings [2,3] and lifetimes of metastable states [4,5], with unprecedented precision. Once the densities of the trapped molecules become high enough and their temperatures become low enough, the intermolecular interactions are anticipated to enable interesting new studies and applications [6,7]. For all these studies it is not only of importance to increase the phase-space density of the trapped molecules but also to increase the time during which the molecules can stay confined in the trap, i.e., to reduce the trap loss processes. Neutral polar molecules in low-field seeking states are routinely trapped in magnetostatic or electrostatic traps by exploiting the Zeeman or Stark effect, respectively $[8,9]$. These traps typically exhibit zero field at the trap center. Within a certain area around the trap center, the trapped molecules can undergo nonadiabatic transitions, widely also referred to as spin flip or Majorana transitions, from a trapped state into a state in which the molecules are no longer trapped. In atomic physics, nonadiabatic transitions in quadrupole magnetic traps seriously hindered the generation of the first Bose-Einstein condensates, as these spin flips made it impossible to reach the required ultracold regime $[10,11]$. The trap losses associated with the presence of the zero field at the trap center were eliminated by implementing the time-averaged orbiting potential (TOP) trap on the one hand [12] and by keeping the atoms away from the trap center with an optical plug on the other hand [13]. Generally, trap loss due to nonadiabatic transitions can be completely suppressed by creating a nonzero field minimum in the trap center. Already in 1962, Ioffe introduced a special variant of a magnetostatic trap with a field offset in the center for nuclear physics experiments [14]. Pritchard [15] suggested in 1983 to use such a trap for the confinement of neutral atoms. This type of magnetostatic trap is now widely known as the Ioffe-Pritchard (IP) trap.

\footnotetext{
*Corresponding author; schnell@fhi-berlin.mpg.de
}

For trapped polar molecules, losses due to nonadiabatic transitions have not been experimentally reported upon yet. Nevertheless, several possible geometries for an electrostatic analog of an IP-type trap have been suggested to prevent these possible losses, such as a chain-linked trap [16] or a six-wire trap [17]. More recently, a trap with an electric field offset in the center has been demonstrated for Rydberg atoms [18]. Theoretical studies on the loss of molecules from purely electrostatic traps [19] or, more generally, from magnetoelectrostatic traps [20] due to nonadiabatic transitions have also recently appeared. In both studies it was concluded that-although this can be different for any particular molecule - the loss rate is negligible at the millikelvin temperatures that are currently achieved in electrostatic traps.

In this Rapid Communication, we experimentally study trap losses due to nonadiabatic transitions in electrostatically trapped ammonia molecules. We quantify this trap loss mechanism from measurements of the trapping times in an IP-type electrostatic trap with either a zero field or an offset field at the center under otherwise identical conditions. Moreover, we measured the trapping times for three isotopologues of ammonia, namely, ${ }^{14} \mathrm{NH}_{3},{ }^{14} \mathrm{ND}_{3}$, and ${ }^{15} \mathrm{ND}_{3}$. These molecules have subtle differences in their energy level structure, resulting in different probabilities for nonadiabatic transitions. Contrary to the theoretical expectations, the results demonstrate the enormous importance of trap losses due to nonadiabatic transitions even at the present $30 \mathrm{mK}$ temperatures.

Figure 1(a) shows the energy of the $|J, K\rangle=|1,1\rangle$ level of $\mathrm{NH}_{3}$ and $\mathrm{ND}_{3}$ in electric fields of up to $150 \mathrm{kV} / \mathrm{cm}$. The upper (lower) inversion doublet components of this level are seen to split into a low-field (high-field) seeking set of hyperfine levels labeled $M K=-1(M K=+1)$ and into a set of hyperfine levels labeled $M K=0$ that are basically not shifted in the fields. The zero-field inversion splitting of ${ }^{14} \mathrm{NH}_{3}$ with $W_{i n v}=23.7 \mathrm{GHz}$ is considerably larger than the $W_{i n v}=1.59 \mathrm{GHz}$ of ${ }^{14} \mathrm{ND}_{3}$. At low electric fields, the Stark shift of the $M K=-1$ set of levels is proportional to $E^{2} / W_{i n v}$ and is therefore considerably less for ${ }^{14} \mathrm{NH}_{3}$ than for ${ }^{14} \mathrm{ND}_{3}$ in a given electric field with magnitude $E$. In Fig. 1(b) the Stark shift of the upper inversion doublet component of ${ }^{14} \mathrm{NH}_{3}$ is shown for low electric fields on an expanded scale 
(a)
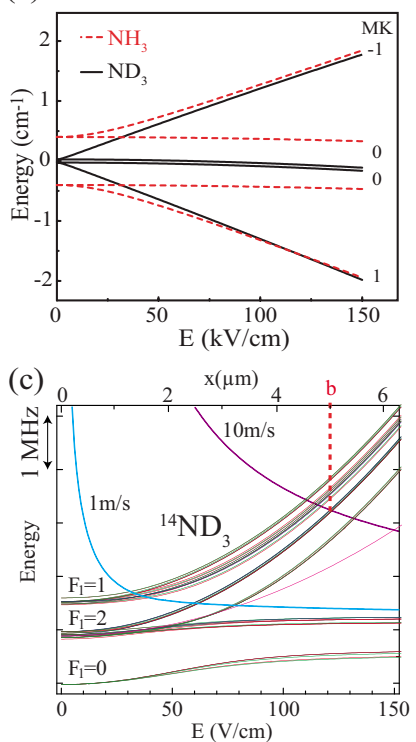

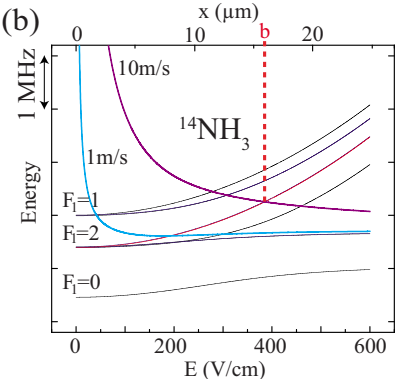

(d)

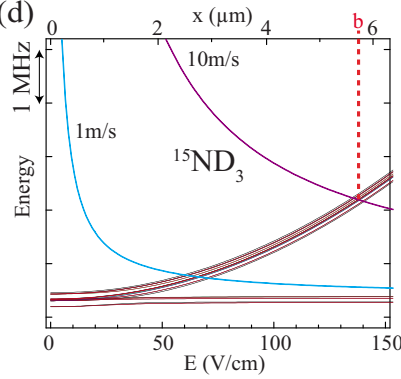

FIG. 1. (Color online) (a) Energy of the $|J, K\rangle=|1,1\rangle$ level of ${ }^{14} \mathrm{NH}_{3}$ (dashed line) and ${ }^{14} \mathrm{ND}_{3}$ (straight line) in fields up to 150 $\mathrm{kV} / \mathrm{cm}$. In panel (b), (c), and (d) the Stark shift of the upper inversion doublet hyperfine components in low electric fields is shown on expanded scales for ${ }^{14} \mathrm{NH}_{3},{ }^{14} \mathrm{ND}_{3}$, and ${ }^{15} \mathrm{ND}_{3}$, respectively. For the calculation of the electric field dependence of the hyperfine components, see Ref. [22]. The $v / x$ curves indicate the rate of change in the direction of the electric field for a molecule with velocity $v(1$ and $10 \mathrm{~m} / \mathrm{s})$ passing the trap center at a distance $x$ (upper axis).

such that the individual hyperfine levels can be recognized. The quadrupole interaction of the ${ }^{14} \mathrm{~N}$ nucleus causes the observed main splitting in three groups of levels, labeled with the quantum number $F_{1}[21]$. The nuclear spins of the hydrogen atoms cause small additional splittings. In the electric field, the overall splitting behavior into (almost) unshifted levels belonging to $M K=0$ and levels that shift quadratically with $E$, belonging to $M K=-1$, is recognized. The individual hyperfine levels have a definite angular momentum projection quantum number $M_{F}$, where $\vec{F}$ is the total angular momentum. As the electric field scales linearly with the distance from the center in a quadrupole electrostatic trap, a distance scale $(x$ in $\mu \mathrm{m})$ appropriate for the trap used in our experiment is indicated on top of the figure as well. In the lower two panels of Fig. 1 the behavior of the hyperfine levels of ${ }^{14} \mathrm{ND}_{3}$ and ${ }^{15} \mathrm{ND}_{3}$ in electric fields is shown [22]. Note that the electric field scale, and thereby the distance scale, is now a factor 4 smaller than for ${ }^{14} \mathrm{NH}_{3}$. For ${ }^{14} \mathrm{ND}_{3}$ and ${ }^{15} \mathrm{ND}_{3}$, the magnitude of the inversion splitting is quite similar (1.59 and $1.43 \mathrm{GHz}$, respectively) but their hyperfine structure is rather different. As the deuterium atoms have a nuclear spin of one, the hyperfine structure in the deuterated isotopologues of ammonia is generally quite complicated. In ${ }^{14} \mathrm{ND}_{3}$, the hyperfine levels again split in three groups of levels due to the quadrupole coupling of the ${ }^{14} \mathrm{~N}$ nucleus [22]. The ${ }^{15} \mathrm{~N}$ nucleus has no quadrupole moment, resulting in less (11 instead of 16 for ${ }^{14} \mathrm{ND}_{3}$ ) hyperfine levels, but these are closer spaced and without a clear substructure for ${ }^{15} \mathrm{ND}_{3}[2]$.

Polar molecules are oriented by the local electric fields inside the trap, and they normally remain in the same quantum state and adiabatically follow the field while moving through the trap. The adiabatic eigenstates can be quantized with respect to the axis along the electric field vector $\vec{E}$ and can thus be assigned the quantum number $M_{F}$. Nonadiabatic transitions can occur when the molecules cannot follow the rapid change of the direction of the electric field when they pass with velocity $v$ at a close distance $x$ to the region of zero electric field at the trap center. When this rate of change, given by $v / x$, is larger than the energy difference between levels in the molecule, nonadiabatic transitions can occur. As $\vec{v}$ is in general not oriented along $\vec{E}$, nonadiabatic transitions with $\Delta M_{F}=0$ as well as with $\Delta M_{F}= \pm 1$ will be possible. In the case of ammonia, nonadiabatic transitions from the $M K=-1$ to the $M K=0$ set of hyperfine levels will cause trap loss. The frequency of these transitions at a distance $x$ from the trap center is proportional to $x^{2} / W_{i n v}$. From this it is seen that nonadiabatic transitions can occur when the molecules come closer to the trap center than a typical distance $b \propto\left(W_{i n v} v\right)^{1 / 3}$. In Figs. 1(b)-1(d) curves of $v / x$ are indicated (in $\mathrm{MHz}$ ) for two different velocities $(v=1$ and $10 \mathrm{~m} / \mathrm{s})$ relative to the highest frequency $M K=0$ hyperfine level. The crossing point of these curves with each of the $M K=-1$ hyperfine levels gives the corresponding distance $b$ for nonadiabatic losses from that particular hyperfine level. Obviously, when there is a large nonzero field at the center of the trap, nonadiabatic transitions from the $M K=-1$ to the $M K=0$ levels will no longer be possible. Nonadiabatic transitions within the $M K=-1$ set of hyperfine levels can still occur to some extent, although also these will be largely suppressed as only the magnitude of the electric field, but no longer the direction, changes rapidly when the molecules pass through the center of the trap.

In the experiment (vide infra), we detect molecules in all low-field seeking $M K=-1$ hyperfine levels simultaneously. It is evident from inspection of the Stark curves shown in Fig. 1 that each of these levels will have a different nonadiabatic transition rate. Therefore, when the trap loss is dominated by nonadiabatic transitions, a multiexponential decay is expected to be observed. The rate for nonadiabatic transitions depends on how often the molecule passes through the volume with cross section $b^{2}$ near the center of the trap. This rate can be approximated by $f_{o s c}\left(b / b_{0}\right)^{2}$, where $f_{\text {osc }}$ is the oscillation frequency of the molecules in the trap and $b_{0}$ is the mean value of the impact parameter of the trajectories of the molecules. With a typical value for $f_{\text {osc }}$ of $1-2 \mathrm{kHz}$ and with $b_{0}$ on the order of $0.2 \mathrm{~mm}$, a value of $b$ around $5 \mu \mathrm{m}$ will lead to a loss rate due to nonadiabatic transitions on the order of $1 \mathrm{~Hz}$.

The experimental setup, together with an expanded view of the IP-type electrostatic trap, is schematically shown in Fig. 2(a). A detailed description of the molecular beam machine and in particular of the deceleration of a beam of ammonia molecules is given elsewhere [23]. Decelerated packets of either ${ }^{14} \mathrm{NH}_{3},{ }^{14} \mathrm{ND}_{3}$, or ${ }^{15} \mathrm{ND}_{3}$ molecules leave the decelerator in the low-field seeking $M K=-1$ hyperfine levels of the $|J, K\rangle=|1,1\rangle$ level with a mean velocity of around 20 $\mathrm{m} / \mathrm{s}$ and with a full width at half maximum (FWHM) veloc- 
(a)

(b)

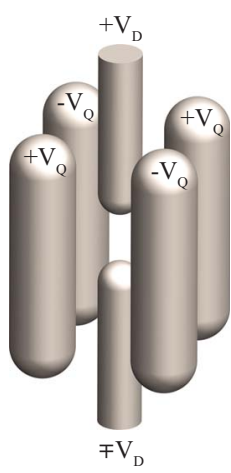

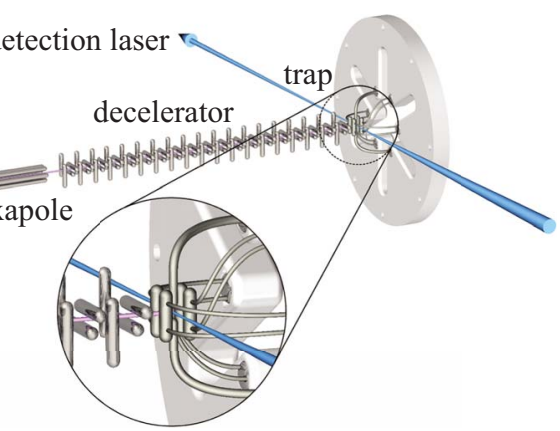

(c)

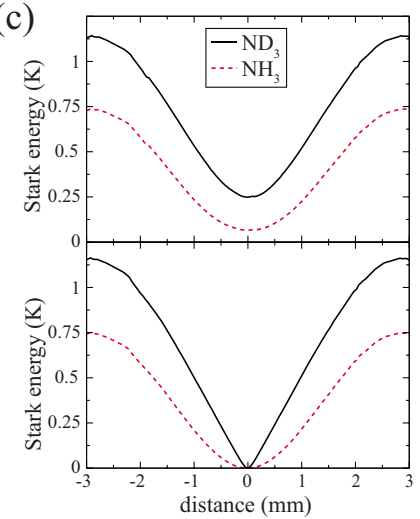

FIG. 2. (Color online) Scheme of the experimental setup, with an expanded view of the IP-type electrostatic trap. The voltages applied to the six cylindrical electrodes are shown (b), together with the resulting potential energies for $\mathrm{NH}_{3}$ (dashed line) and $\mathrm{ND}_{3}$ (straight line) along a line in the horizontal symmetry plane for a nonzero (top) and a zero (bottom) electric field at the trap center (c).

ity spread of about $10 \mathrm{~m} / \mathrm{s}$. The decelerated packet contains approximately $10^{5}-10^{6}$ molecules and has a spatial extent of about $2 \mathrm{~mm}$ along the molecular beam axis and is about $2 \times 2 \mathrm{~mm}^{2}$ in the transverse direction at the exit of the decelerator [23]. The molecules are decelerated to a mean velocity around $0 \mathrm{~m} / \mathrm{s}$ upon entering the trap, which is centered $8 \mathrm{~mm}$ behind the decelerator. After a certain trapping time, the trap is turned off and the molecules are state-selectively detected using a laser based ionization detection scheme.

The trap consists of six cylindrical electrodes, shown more clearly in Fig. 2(b). To the four outer electrodes $(3 \mathrm{~mm}$ diameter) positive and negative voltages $\left( \pm V_{Q}\right)$ are applied such as to generate a quadrupole field. The two electrodes (2 $\mathrm{mm}$ diameter) centered on the symmetry axis of the trap enable to create an additional dipole field. When voltages of opposite polarity $\left( \pm V_{D}\right)$ are applied to these electrodes, a nonzero electric field is generated at the center of the trap; by applying the same polarity $\left(+V_{D}\right)$ to the center electrodes, a standard quadrupole trap with a zero field at the center is obtained. In Fig. 2(c) the potential energy for the $M K=-1$ levels of $\mathrm{NH}_{3}$ and $\mathrm{ND}_{3}$ in the trap is shown along a line in the horizontal symmetry plane both for a zero (bottom) and a nonzero field (top) at the trap center. In the latter case, the trap depth is actually limited by the occurrence of four saddle points of the electric fields in between the electrodes. For $V_{Q}=10 \mathrm{kV}$ and $V_{D}=3 \mathrm{kV}$, the offset electric field at the center is $16 \mathrm{kV} / \mathrm{cm}$ and the trap depths are about $290 \mathrm{mK}$ for $\mathrm{ND}_{3}$ and $130 \mathrm{mK}$ for $\mathrm{NH}_{3}$. With no electric field at the cen-
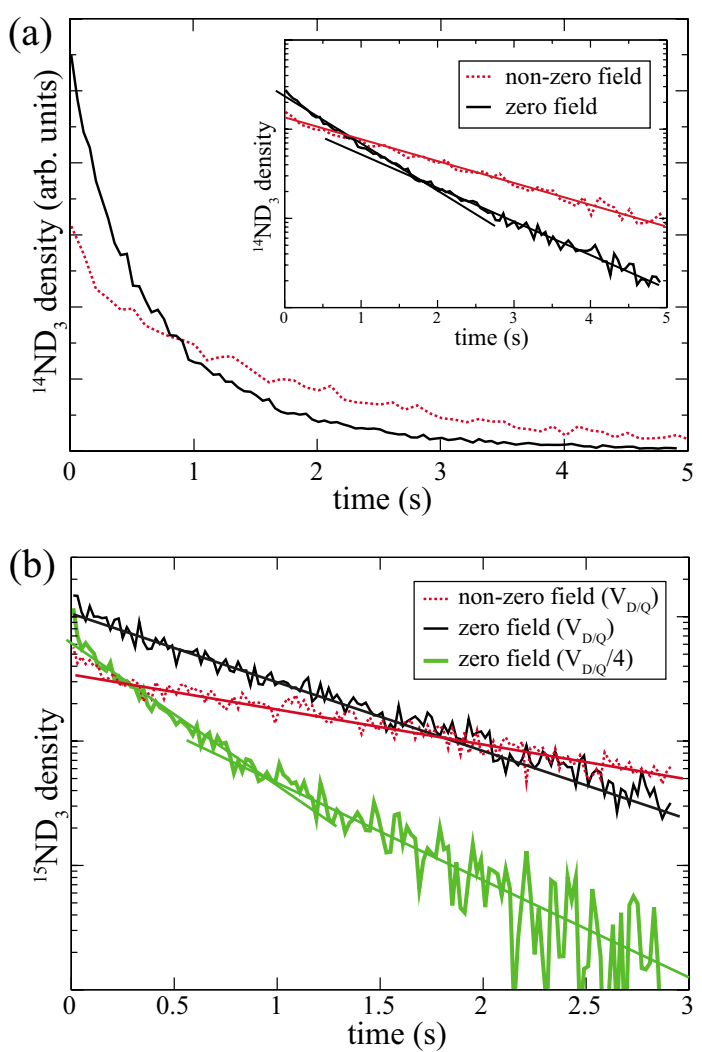

FIG. 3. (Color online) (a) Lifetime measurements for ${ }^{14} \mathrm{ND}_{3}$ in the two trap configurations, both on a linear and on a logarithmic scale (inset). In the inset, straight lines are included to guide the eyes. (b) Lifetime measurements for ${ }^{15} \mathrm{ND}_{3}$ with original $\left(V_{D / Q}\right)$ and reduced $\left(V_{D / Q} / 4\right)$ trap voltages on a logarithmic scale. The measurements have been performed with intervals of $20 \mathrm{~ms}$ and with 16 averages per time point. The nonzero field in the trap center is 16 $\mathrm{kV} / \mathrm{cm}$ for all measurements shown.

ter, the traps are considerably deeper, about $1.2 \mathrm{~K}$ for $\mathrm{ND}_{3}$ and $750 \mathrm{mK}$ for $\mathrm{NH}_{3}$.

Figure 3(a) shows measurements of the density of ${ }^{14} \mathrm{ND}_{3}$ molecules in the electrostatic trap as a function of the time during which the trap has been switched on, both for zero and nonzero fields at the center. Although the number density in the zero-field trap is originally higher due to a better trap loading, it is also seen to decay significantly faster. In both cases, the trap is considerably deeper than the temperature of the trapped ensemble of molecules; the latter has been determined from expansion measurements to be around $30 \mathrm{mK}$. The molecules can therefore only leave the trap via collisions, via optical pumping due to blackbody radiation, or via nonadiabatic transitions. Losses due to collisions can either originate from (in)elastic collisions of the trapped molecules with background gas or from cold collisions among the trapped molecules. At the present densities in the trap $\left(10^{7}-10^{8} \mathrm{~cm}^{-3}\right)$, collisions of the trapped molecules with each other can be neglected. We have experimentally verified this by changing the number density of trapped molecules over 1 order of magnitude, without observing any change in the trapping time. The ${ }^{14} \mathrm{ND}_{3}$ molecules in the low-field seeking $M K=-1$ hyperfine levels can be optically pumped to excited rovibrational levels due to blackbody radiation from 
the room-temperature environment, leading to a calculated finite lifetime of about $7 \mathrm{~s}$ [24]. The purely single exponential decay of the number of ${ }^{14} \mathrm{ND}_{3}$ molecules in the trap with the nonzero field has a $1 / e$ time constant of $1.9 \mathrm{~s}$. This decay time results from the combination of optical pumping due to blackbody radiation and (in)elastic collisions with background gas (background pressure of $10^{-8} \mathrm{mbar}$ ). By simply changing the polarity on one of the center electrodes of the trap, under otherwise identical conditions, a much faster and multiexponential decay is observed [Fig. 3(a)]; the additional trap loss observed in the trap with zero electric field at the center is solely due to nonadiabatic transitions. From these measurements, it appears that the nonadiabatic transitions for ${ }^{14} \mathrm{ND}_{3}$ can be approximated with only two time constants. Referring to Fig. 1(c), the fast decay can be attributed to nonadiabatic transitions within the $F_{1}=2$ set of hyperfine levels, whereas the slow decay can be attributed to nonadiabatic transitions from the $F_{1}=1$ to the $F_{1}=2$ set of hyperfine levels. When the nonadiabatic transitions are modeled in this way (with the prefactors for the two exponentials determined by the number of $M_{F}$ levels), time constants of about 0.25 and $2.0 \mathrm{~s}$, respectively, are extracted for these processes. The errors on these $1 / e$ time constants are less than $20 \mathrm{~ms}$.

We have performed a similar series of experiments for ${ }^{14} \mathrm{NH}_{3}$ (data not shown). In the zero-field trap, a biexponential decay is also observed for ${ }^{14} \mathrm{NH}_{3}$, which can be explained in the same way as for ${ }^{14} \mathrm{ND}_{3}$. After correction for the overall decay as measured for ${ }^{14} \mathrm{NH}_{3}$ in the trap with the offset field, the time constants for the fast and the slow processes are determined as 0.13 and $3.5 \mathrm{~s}$. The faster initial decay can be explained by the larger value of $b$ whereas the slower decay from the $F_{1}=1$ to the $F_{1}=2$ set of hyperfine levels than for ${ }^{14} \mathrm{ND}_{3}$ can be rationalized by the slightly larger energetic separation and the reduced number of levels of ${ }^{14} \mathrm{NH}_{3}$.

In Fig. 3(b), the density of ${ }^{15} \mathrm{ND}_{3}$ molecules in the trap is shown as a function of time. In this case, the decay in the zero-field trap is seen to be almost single exponential, and the $1 / e$ time constant due to the nonadiabatic transitions is $1.2 \mathrm{~s}$. A more pure single exponential behavior can indeed be expected as all low-field seeking hyperfine levels behave very similar in the electric field [see Fig. 1(d)]. When all voltages on the trap (and thus also the electric field offset in the nonzero field trap) are reduced by a factor of 4 , the lifetime in the trap with the offset electric field stays the same (data not shown). In the zero-field trap, however, the decay is not only faster due to the larger value of $b$ but it is also multiexponential as the grouping of the hyperfine levels is now relatively more important.

As evidenced by the experimental results presented in this work, nonadiabatic transitions can be a dominant loss channel in electrostatically trapped molecules even at temperatures of tens of millikelvins. These trap losses can be effectively prevented by using an electrostatic trap with a nonzero electric field at the center or, for instance, by orbiting the molecules off center in a storage ring [25]. The importance of trap losses due to nonadiabatic transitions depends on the detailed energy level structure of the molecule under consideration. Contrary to the situation in magnetic fields, there is not necessarily a degeneracy of low-field and high-field seeking levels for molecules at zero electric field. As a consequence, molecules in levels that are exclusively low-field seeking, such as ${ }^{14} \mathrm{NH}_{3}$ and ${ }^{14} \mathrm{ND}_{3}$ molecules in the $F_{1}=1$ hyperfine manifold of the $|J, K\rangle=|1,1\rangle$ level [see Figs. 1(b) and $1(\mathrm{c})]$, can be rather immune to nonadiabatic transitions. For some applications it might not be desirable to introduce additional offset fields. In that case it might be possible to select another isotopologue with a slightly different hyperfine structure to effectively suppress the losses due to nonadiabatic transitions.

We thank Henrik Haak for the trap design and for technical support and Steven Hoekstra and Andreas Osterwalder for fruitful discussions. M.S. acknowledges a Liebig grant from the Fonds der Chemischen Industrie.
[1] S. Y. T. van de Meerakker, H. L. Bethlem, and G. Meijer, Nat. Phys. 4, 595 (2008).

[2] J. van Veldhoven et al., Eur. Phys. J. D 31, 337 (2004).

[3] E. R. Hudson, H. J. Lewandowski, B. C. Sawyer, and J. Ye, Phys. Rev. Lett. 96, 143004 (2006).

[4] S. Y. T. van de Meerakker, N. Vanhaecke, M. P. J. van der Loo, G. C. Groenenboom, and G. Meijer, Phys. Rev. Lett. 95, 013003 (2005).

[5] W. C. Campbell et al., Phys. Rev. Lett. 100, 083003 (2008).

[6] R. V. Krems, Int. Rev. Phys. Chem. 24, 99 (2005).

[7] A. André et al., Nat. Phys. 2, 636 (2006).

[8] J. D. Weinstein et al., Nature (London) 395, 148 (1998).

[9] H. L. Bethlem et al., Nature (London) 406, 491 (2000).

[10] W. Ketterle, Rev. Mod. Phys. 74, 1131 (2002).

[11] E. A. Cornell and C. E. Wieman, Rev. Mod. Phys. 74, 875 (2002).

[12] W. Petrich, M. H. Anderson, J. R. Ensher, and E. A. Cornell, Phys. Rev. Lett. 74, 3352 (1995).
[13] K. B. Davis et al., Phys. Rev. Lett. 75, 3969 (1995).

[14] Y. V. Gott, M. S. Ioffe, and V. G. Telkovsky, Nucl. Fusion 3, 1045 (1962).

[15] D. E. Pritchard, Phys. Rev. Lett. 51, 1336 (1983).

[16] N. E. Shafer-Ray, K. A. Milton, B. R. Furneaux, E. R. I. Abraham, and G. R. Kalbfleisch, Phys. Rev. A 67, 045401 (2003).

[17] G. Xu, Ph.D. thesis, The University of Texas at Austin, 2001.

[18] S. Hogan and F. Merkt, Phys. Rev. Lett. 100, 043001 (2008).

[19] M. Kajita, Eur. Phys. J. D 38, 315 (2006).

[20] M. Lara, B. L. Lev, and J. L. Bohn, Phys. Rev. A 78, 033433 (2008).

[21] S. G. Kukolich, Phys. Rev. 156, 83 (1967).

[22] J. van Veldhoven, R. T. Jongma, B. Sartakov, W. A. Bongers, and G. Meijer, Phys. Rev. A 66, 032501 (2002).

[23] H. L. Bethlem et al., Phys. Rev. A 65, 053416 (2002).

[24] S. Hoekstra et al., Phys. Rev. Lett. 98, 133001 (2007).

[25] F. M. H. Crompvoets et al., Nature (London) 411, 174 (2001). 